\title{
Clinical utility of ixekizumab in the treatment of moderate-to-severe plaque psoriasis
}

This article was published in the following Dove Press journal:

Psoriasis: Targets and Therapy

16 November 2017

Number of times this article has been viewed

\section{Sahil Sekhon \\ Caleb Jeon \\ Mio Nakamura \\ Di Yan \\ Ladan Afifi \\ Tina Bhutani \\ Ethan Levin}

Department of Dermatology, University of California, San Francisco, San Francisco, CA, USA
Correspondence: Sahil Sekhon Department of Dermatology, University of California San Francisco, 515 Spruce Street, San Francisco, CA 94I18, USA $\mathrm{Tel}+\mathrm{I} 4154764019$

Fax +I 4155024126

Email sahilsekhon@gmail.com
Abstract: Psoriasis vulgaris is a chronic, immune-mediated systemic disease that affects $\sim 7.5$ million people in the US. It can be treated with many therapies, often in combination, which include topicals, phototherapy, oral systemics, and biologics. Biologic agents target specific components of the immune system involved in the pathogenesis of psoriasis including TNF-alpha, IL-12, IL-17, and IL-23. The biologic ixekizumab, approved for the treatment of moderate-severe plaque psoriasis in the US, targets IL-17. This review describes the role of IL-17 in psoriasis, the mechanism by which ixekizumab targets this cytokine, and the clinical utility of ixekizumab. Keywords: ixekizumab, biologics, psoriasis, IL-17, anti-IL-17

\section{Introduction}

Psoriasis vulgaris is a chronic, immune-mediated systemic disease that affects multiple organ systems in the body. Although most visible on the skin, psoriasis can also be associated with comorbidities involving the cardiovascular and musculoskeletal systems. Psoriasis vulgaris, "classically characterized by thickened, red areas of skin covered with silvery scales," affects $\sim 7.5$ million people in the US and has a prevalence of up to $11.4 \%$ in some countries. ${ }^{1,2}$ The disease can present as a single plaque (localized disease) or involve nearly all body surfaces (generalized disease). It has many morphologic variants, including guttate, plaque, palmoplantar, and erythrodermic psoriasis. Of these, plaque psoriasis is the most common, with severity ranging between mild, moderate, and severe, with overlap between these categories (e.g., mild-moderate and moderate-severe disease). Treatment for psoriasis tends to include combinations of various therapies, including topicals, phototherapy, oral systemics, and biologics. Biologic therapies target specific components of the immune system involved in the pathogenesis of psoriasis and currently include agents that target TNF-alpha, IL-12, IL-17, and IL-23. The focus of this review is one such biologic agent, ixekizumab, that targets IL-17 and has been approved for use in the treatment of moderate-severe plaque psoriasis by the US Food and Drug Administration (FDA) since $2016 .{ }^{3}$

\section{Role of IL-I 7 in psoriasis vulgaris}

Psoriasis is a T-cell-mediated disease, involving complex interactions between various cell types and cytokines. A subset of CD4+ T cells called helper T cells or Th cells of various lineages play a role in the pathogenesis of psoriasis. Th17 cells, one of these cell lines, are part of the Th17 axis that plays a key role in the pathogenesis of psoriasis. ${ }^{4}$ 
In psoriasis, activated dendritic cells and macrophages produce IL-23, which causes differentiation of naïve T cells into Th17 cells. ${ }^{5}$ Th17 cells produce many cytokines including IL-17 and IL-22, which cause, among other things, keratinocyte proliferation and production of proinflammatory cytokines and chemokines, which then create a self-amplifying loop that leads to perpetuation of skin inflammation. ${ }^{6}$ IL-17 is one of the key cytokines involved in Th17 signaling..$^{7-10}$ The IL-17 cytokine family includes the six homodimer subtypes (IL-17A to IL-17F), the heterodimer IL-17A/F, and five receptors (IL-17RA to IL17-RE). ${ }^{11,12}$ Th17 cells secrete only three of the six IL-17 cytokines (IL-17A, IL-17F, and IL-17A/F). Of these, IL-17A is one of the most important in the pathogenesis of psoriasis and is targeted by Taltz (ixekizumab). ${ }^{13}$

\section{Ixekizumab}

Ixekizumab was approved by the FDA in 2016 for the treatment of moderate-to-severe plaque psoriasis. It is a humanized monoclonal IgG subclass 4-kappa (IgG4- $\kappa)$ antibody that neutralizes interleukin-17A homodimers and IL-17A/F heterodimers. Treatment with ixekizumab results in decreased expression of interferon- $\gamma$, IL-17, IL-22, IL-23, and other cytokines important for the pathogenesis of psoriasis. As a result, keratinocyte proliferation and neutrophil migration to the epidermis is slowed. ${ }^{14}$ Ixekizumab is subcutaneously administered with induction and maintenance dosing regimens. The induction phase consists of an initial dose of $160 \mathrm{mg}$ delivered on week 0 , followed by $80 \mathrm{mg}$ administered every 2 weeks through week 12. After week 12, the maintenance dose of $80 \mathrm{mg}$ is administered every 4 weeks. Ixekizumab is available in both prefilled syringes and auto-injector pens, each containing $80 \mathrm{mg} / \mathrm{mL}$ of ixekizumab. ${ }^{3}$

\section{Efficacy}

\section{Phase III pivotal trials}

Ixekizumab had three pivotal trials: Uncover-1, -2, and $-3 .{ }^{15-17}$ Each of these trials involved a starter dose of $160 \mathrm{mg}$ of ixekizumab administered at week 0 , followed by $80 \mathrm{mg}$ administered every 2 weeks or every 4 weeks.

\section{Trial designs}

Uncover-1 compared the two dosing regimens of ixekizumab to placebo, while Uncover-2 and -3 compared the two ixekizumab regimens to placebo and etanercept dosed at $50 \mathrm{mg}$ twice weekly. After 12 weeks of treatment, patients in the Uncover-1 and -2 trials who had response to ixekizumab, defined as minimal (1) or clear (0) on the static Physician's Global Assessment (sPGA), were rerandomized to receive placebo, ixekizumab $80 \mathrm{mg}$ every 4 weeks, or ixekizumab $80 \mathrm{mg}$ every 12 weeks through week 60 . Patients who had sPGA >1 at week 12 were started on ixekizumab $80 \mathrm{mg}$ every 4 weeks. Patients who relapsed between weeks 12 and 60 , which was defined as an sPGA score of $\geq 3$, were either continued on ixekizumab $80 \mathrm{mg}$ every 4 weeks or started on ixekizumab $80 \mathrm{mg}$ every 4 weeks. Patients who received placebo in the initial 12 weeks of the study and achieved sPGA 0 or 1 were started on placebo treatments every 4 weeks until they experienced relapse, after which they were treated with ixekizumab. In Uncover-2, patients who were treated with etanercept and had sPGA $>1$ at week 12 were started on ixekizumab $80 \mathrm{mg}$ every 4 weeks after a 4-week washout period. Patients who received etanercept in the initial 12 weeks of the study and achieved sPGA 0 or 1 were started on placebo treatments every 4 weeks until they experienced relapse, after which they were treated with ixekizumab. In the Uncover-3 trial, patients entered a longterm extension period after week 12 and received $80 \mathrm{mg}$ of ixekizumab every 4 weeks through week 60 . The co-primary end points of Uncover-1, -2, and -3 were Psoriasis Area and Severity Index (PASI)-75 and sPGA 0 or 1 at week 12.

\section{Uncover-I}

Uncover-1 enrolled 1296 patients who were randomized in a 1:1:1 ratio to placebo (431 patients), ixekizumab $80 \mathrm{mg}$ every 2 weeks (433 patients), or ixekizumab $80 \mathrm{mg}$ every 4 weeks (432 patients). At week 12, patients treated every 2 and 4 weeks achieved PASI-75, PASI-90, and SPGA at significantly higher rates than patients treated with placebo. In patients treated every 2 weeks, $\sim 89 \%$ achieved PASI-75, $82 \%$ achieved sPGA 0 or 1 , and $71 \%$ achieved PASI- 90 . Table 1 highlights specific PASI-75, PASI-90, and PASI-100 response rates for patients treated every 2 and every 4 weeks with ixekizumab.

Table I PASI response rates at 12 weeks in Uncover- I, -2, and -3 by dosing regimen

\begin{tabular}{llll}
\hline $\begin{array}{l}\text { Study and dosing } \\
\text { regimen }\end{array}$ & $\begin{array}{l}\text { PASI-75 } \\
\text { (\%) }\end{array}$ & $\begin{array}{l}\text { PASI-90 } \\
\text { (\%) }\end{array}$ & $\begin{array}{l}\text { PASI-100 } \\
\text { (\%) }\end{array}$ \\
\hline $\begin{array}{l}\text { Uncover-I } \\
\text { Ixekizumab every 2 weeks }\end{array}$ & 89.1 & 70.9 & 35.3 \\
$\begin{array}{l}\text { Ixekizumab every 4 weeks } \\
\text { Uncover-2 and -3 pooled }\end{array}$ & 82.6 & 64.6 & 33.6 \\
$\begin{array}{l}\text { Ixekizumab every 2 weeks } \\
\text { Ixekizumab every 4 weeks }\end{array}$ & 88.5 & 69.3 & 39.0 \\
\hline
\end{tabular}

Abbreviation: PASI, Psoriasis Area and Severity Index. 
The results from the randomized withdrawal period between weeks 12 and 60 are discussed in detail in the following combined with results from Uncover-2.

\section{Uncover-2}

Uncover-2 enrolled 1224 patients who were randomized in a 2:2:2:1 ratio to ixekizumab $80 \mathrm{mg}$ every 2 weeks (351 patients), ixekizumab $80 \mathrm{mg}$ every 4 weeks ( 347 patients), etanercept $50 \mathrm{mg}$ twice weekly (358 patients), and placebo (168 patients). After 12 weeks of treatment, a significantly greater proportion of patients in both ixekizumab treatment groups achieved PASI-75 and sPGA 0 or 1 compared to placebo and etanercept. Specifically, $89.7 \%$ and $77.5 \%$ of patients receiving ixekizumab every 2 weeks and every 4 weeks, respectively, achieved PASI-75, which were both significantly greater than the PASI-75 response rate of $41.6 \%$ of the etanercept-treated group. Similarly, sPGA 0 or 1 was achieved by $83.2 \%$ and $72.9 \%$ of patients treated with ixekizumab every 2 and 4 weeks, respectively, while only $36.0 \%$ of patients treated with etanercept achieved sPGA 0 or 1 after 12 weeks of treatment. At week 12, PASI-90 and PASI-100 response rates in patients receiving ixekizumab every 2 weeks were $70.7 \%$ and $40.5 \%$, respectively. In patients treated every 4 weeks with ixekizumab, PASI90 and PASI-100 response rates were $59.7 \%$ and $30.8 \%$ at week 12 , respectively. These results are also listed in Table 1.

The results from the randomized withdrawal period between weeks 12 and 60 are discussed in detail in the following.

\section{Uncover-3}

Uncover-3 enrolled 1346 patients in total in a 2:2:2:1 ratio similar to the Uncover-2 trial. In all, 385 patients received ixekizumab $80 \mathrm{mg}$ every 2 weeks, 386 patients received ixekizumab $80 \mathrm{mg}$ every 4 weeks, 382 patients received etanercept $50 \mathrm{mg}$ twice weekly, and 193 patients received placebo. Patients treated with ixekizumab $80 \mathrm{mg}$ administered every 2 and every 4 weeks experienced significantly greater PASI- 75 response rates $(87.3 \%$ and $84.2 \%$, respectively) compared to patients treated with etanercept (53.4\%). Similarly, rates of patients achieving sPGA 0 or 1 were significantly greater in patients treated with ixekizumab every 2 weeks and every 4 weeks $(80.5 \%$ and $75.4 \%$, respectively) when compared to patients treated with etanercept $50 \mathrm{mg}$ twice weekly (41.6\%). PASI-90 response rates at week 12 were $68.1 \%$ (ixekizumab every 2 weeks) and $65.3 \%$ (ixekizumab every 4 weeks), and PASI-100 response rates were $37.7 \%$ (ixekizumab every 2 weeks) and 35.0\% (ixekizumab every 4 weeks) at that same time point.

After 12 weeks, patients were enrolled in a long-term extension period at the discretion of investigators and received ixekizumab $80 \mathrm{mg}$ every 4 weeks for up to 264 weeks. ${ }^{15}$

\section{Randomized withdrawal period (Uncover-I and -2)}

In the randomized withdrawal period (weeks 12-60), the percentage of patients receiving ixekizumab every 4 weeks who maintained sPGA 0 or 1 at week 60 was $73.8 \% .{ }^{15}$ Only $39.0 \%$ of patients who received ixekizumab every 12 weeks maintained sPGA 0 or 1 , while just $7.0 \%$ of patients who received placebo maintained sPGA 0 or 1 . Superior efficacy was shown in patients treated with ixekizumab every 2 weeks for the first 12 weeks followed by every 4 weeks from week 12 to 60 versus patients treated with ixekizumab every 4 weeks from week 0 to 60 (sPGA: 78.3 vs 68.7 , PASI-90: $76.5 \%$ vs $66.7 \%$, PASI-100: $57.5 \%$ vs $49.7 \%$, respectively). PASI response rates at 60 weeks are listed in Table 2, stratified by induction dosing regimen.

During the withdrawal phase, the average time to loss of response after switching to placebo was 169 days for patients originally treated with ixekizumab every 2 weeks and 148 days in those originally treated every 4 weeks.

Patients who experienced relapse after withdrawal of treatment (i.e., randomized to receive placebo) were retreated with ixekizumab $80 \mathrm{mg}$ every 4 weeks and were followed through 24 weeks of retreatment. Table 3 details the

Table 2 PASI response rates at week 60 in week 12 responders in Uncover-I and -2 by induction and maintenance dosing

\begin{tabular}{lll}
\hline $\begin{array}{l}\text { Dosing regimen, induction } \rightarrow \\
\text { maintenance }\end{array}$ & $\begin{array}{l}\text { PASI-90 } \\
\text { (\%) }\end{array}$ & $\begin{array}{l}\text { PASI- I00 } \\
\text { (\%) }\end{array}$ \\
\hline Every 2 weeks $\rightarrow$ every I 2 weeks & 40.6 & 18.9 \\
Every 2 weeks $\rightarrow$ every 4 weeks & 76.5 & 57.5 \\
Every 4 weeks $\rightarrow$ every I2 weeks & 34.7 & 20.9 \\
Every 4 weeks $\rightarrow$ every 4 weeks & 66.7 & 49.7 \\
\hline
\end{tabular}

Abbreviation: PASI, Psoriasis Area and Severity Index.

Table 3 Recapture rates after retreatment stratified by induction dosing regimen for the first 12 weeks in randomized withdrawal period of Uncover-I and -2

\begin{tabular}{lll}
\hline $\begin{array}{l}\text { Outcome } \\
\text { measures }\end{array}$ & $\begin{array}{l}\text { Induction with every } \\
\text { 2-week dosing (\%) }\end{array}$ & $\begin{array}{l}\text { Induction with every } \\
\text { 4-week dosing (\%) }\end{array}$ \\
\hline sPGA 0 or I & 70.7 & 82.3 \\
PASI-75 & 87.0 & 95.1 \\
PASI-90 & 63.4 & 78.4 \\
PASI-100 & 31.7 & 45.1 \\
\hline
\end{tabular}

Abbreviations: sPGA, static Physician's Global Assessment; PASI, Psoriasis Area and Severity Index. 
recapture of sPGA of 0 or 1, PASI-75, PASI-90, and PASI-100 responses. ${ }^{18}$ Overall, most patients did recapture therapeutic response with retreatment, but when compared to patients with continuous treatment, efficacy was decreased in patients with interrupted treatment. This suggests that ixekizumab should be used to achieve improvement of psoriasis through the induction phase and then continued with the maintenance phase to sustain improvement of psoriasis as opposed to being used for intermittent therapy of psoriasis flare ups. In patients who began retreatment, the median time to achieve PASI-75 response was 4 weeks.

\section{Efficacy by body weight}

The efficacy of ixekizumab in Uncover-1, -2 , and -3 by body weight was evaluated in subgroup analysis with patients divided into three weight categories: $<80 \mathrm{~kg}, 80-<100 \mathrm{~kg}$, and $\geq 100 \mathrm{~kg} .{ }^{19}$ PASI-75 response rates were not meaningfully different between weight groups in patients given ixekizumab every 2 weeks, while there was a trend toward decreasing PASI-75 response rates at higher body weights in patients treated with ixekizumab every 4 weeks. PASI-90 and PASI100 response rates did show decrease in response as body weight increased in both patients treated with ixekizumab every 2 weeks and every 4 weeks. Furthermore, across body weight groups, patients treated every 2 weeks with ixekizumab had greater PASI response rates than patients treated every 4 weeks.

\section{Efficacy in biologic-naïve versus biologic-experienced patients}

Gottlieb et $\mathrm{al}^{20}$ compared the efficacy of ixekizumab in patients with prior biologic exposure to that in patients without prior biologic exposure in the Uncover-2 and -3 trials. In both of these studies, patients who had prior exposure to any IL-17 antagonists or etanercept were excluded, so their evaluation of these subgroups did not include data on patients with these exposures. In these two pivotal trials, $15.5 \%-23.5 \%$ of patients had prior biologic exposure and $45.7 \%-49.5 \%$ of patients had prior exposure to nonbiologic systemic agents. In patients treated with the recommended every 2-week dosing regimen, PASI-75 at week 12 was achieved by $91.5 \%$ of biologic-experienced and $87.7 \%$ of biologic-naïve patients. PASI-90 and PASI-100 response rates were also similar between biologic-experienced and biologic-naïve patients $(67.7 \%$ vs $76.1 \%$ and $37.0 \%$ vs $47.2 \%$, respectively). Patients with prior biologic exposure did not have lower response rates to ixekizumab. Although it appears that patients with prior biologic exposure had slightly better response rates to ixekizumab, this difference was not statistically evaluated, so this conclusion cannot be made. However, it does seem that prior biologic exposure did not affect response to ixekizumab.

\section{Other Phase III studies and studies in special subtypes of psoriasis}

Ixekizumab has been studied in several other non-pivotal clinical trials, including a head-to-head study against ustekinumab in patients with palmoplantar psoriasis, fingernail psoriasis, erythrodermic psoriasis, and generalized palmoplantar pustular psoriasis.

\section{Head-to-head study with ustekinumab}

In a head-to-head study called IXORA-S, patients were randomized to receive either ustekinumab with standard weight-based dosing or ixekizumab at the standard recommended dose. After 12 weeks, ixekizumab-treated patients had significantly higher rates of PASI-90 response, $72.8 \%$ versus $42.2 \%$, and maintained significantly greater PASI response through 24 weeks of treatment. ${ }^{21}$ Patients treated with ixekizumab also had significantly better sPGA and Dermatology Life Quality Index (DLQI) improvement at week 24 .

\section{Palmoplantar psoriasis}

Uncover-1, -2 , and -3 trials included $28 \%$ of patients with non-pustular palmoplantar involvement, with $9.1 \%$ of patients with moderate-to-severe involvement as measured by Palmoplantar Psoriasis Area and Severity Index (PPASI) $\geq 8$. Ixekizumab-treated patients had significantly higher rates of PPASI-100 compared to patients treated with etanercept $(51.8 \%$ vs $32.2 \%)$ after 12 weeks of treatment. ${ }^{22}$

\section{Fingernail psoriasis}

Many patients with psoriasis also have fingernail involvement, which can be both difficult to treat and significantly distressing to patients. In Uncover-3, a subgroup of patients had baseline fingernail involvement, as measured using Nail Psoriasis Severity Index (NAPSI). After 12 weeks of treatment with ixekizumab, the mean percentage improvement was $36.7 \%$ in NAPSI scores and $25.2 \%$ in patients treated with ixekizumab dosed every 4 weeks and every 2 weeks, respectively. At week $60,>50 \%$ of patients had complete resolution of their nail psoriasis, and mean percentage improvement in all patients receiving ixekizumab, regardless of initial treatment (ixekizumab dosed every 2 or 4 weeks, placebo, or etanercept), was $>80 \%$. With the 
recommended dosing regimen for ixekizumab, $62.4 \%$ of patients achieved complete resolution of their nail psoriasis, while mean percentage improvement in NAPSI scores was $83.6 \% .^{23}$

\section{Erythrodermic psoriasis and generalized pustular psoriasis (GPP)}

Ixekizumab was studied in two small groups of patients with erythrodermic psoriasis and GPP as part of the UNCOVER-J clinical trial of ixekizumab in Japan. Erythrodermic patients had $\geq 80 \%$ BSA involvement at screening and baseline, while patients with GPP had to meet the Japanese Ministry of Health, Labor and Welfare criteria for this diagnosis. In total, eight patients with erythrodermic psoriasis and five patients with GPP were enrolled and completed the 52 weeks of the study. At week 12, all eight patients with erythrodermic psoriasis achieved PASI-75, while five patients achieved PASI-90. At this same time point, four patients achieved PASI-75 and three patients achieved PASI-90 from the group of patients with GPP. ${ }^{24}$ At week 52, these same PASI-75 and PASI-90 response rates were maintained in both patient groups, and six of the eight patients in the erythrodermic group achieved sPGA of clear or almost clear, while three of five patients achieved sPGA of clear or almost clear in the GPP group. ${ }^{25}$

\section{Results of special interest from pre-Phase III studies}

In Phase I clinical trials with ixekizumab, 40 patients were treated with varying doses of ixekizumab at 2-week intervals for three doses. At week 16, 10 weeks after their last dose, $74.1 \%$ of patients treated with $150 \mathrm{mg}$ of ixekizumab maintained PASI-90. Furthermore, biopsies of psoriatic skin in patients treated with $50 \mathrm{mg}$ and $150 \mathrm{mg}$ of ixekizumab reflected nearly normal skin by week 6 in terms of keratinocyte proliferation, epidermal hyperplasia, and infiltration by inflammatory cells in the dermis and epidermis. $^{14}$

In a Phase II study, 142 patients were treated with varying doses of ixekizumab. Patients were treated at weeks 0, 2, 4, 8,12 , and $16 .{ }^{26}$ At week 20, patients who received $75 \mathrm{mg}$ and $150 \mathrm{mg}$ of ixekizumab had a significant improvement in scalp psoriasis as measured using the Psoriasis Scalp Severity Index (PSSI) and in their nail psoriasis as measured using the NAPSI. This Phase II study also included an open-label extension (OLE) phase where patients were treated with ixekizumab at a dose of $120 \mathrm{mg}$ every 4 weeks. ${ }^{27}$ By 48 weeks of the OLE study, between $48.7 \%$ and $58.3 \%$ of patients had complete resolution of nail psoriasis lesions. ${ }^{28}$ Patients with scalp psoriasis experienced even greater improvement with ixekizumab, with $77.3 \%-81.3 \%$ of patients experiencing complete resolution of their scalp psoriasis. ${ }^{28}$

\section{Improvement in quality of life (QoL)}

The Uncover trials evaluated QoL improvement with ixekizumab using the validated DLQI. Uncover-2 and -3 showed significantly greater improvement in DLQI scores compared to both placebo and etanercept-treated groups at 12 weeks of treatment. Improvement in DLQI scores was positively correlated with improvement in PASI. Patients with PASI-90 response were more likely to have DLQI scores of 0 or 1 compared to patients who achieved PASI-75. In all, $64.1 \%$ and $64.7 \%$ of patients treated with ixekizumab every 2 weeks achieved DLQI scores of 0 or $1 .{ }^{17}$ After a median of 2.1 weeks, $50 \%$ of patients treated with ixekizumab achieved at least a 5-point reduction in DLQI compared to 4 weeks for patients treated with etanercept. After just 2 weeks of treatment with ixekizumab, a significantly higher percentage of patients treated with ixekizumab reported DLQI scores of 0 or 1 compared to placebo and etanercept (17.9\% and $18.8 \%$ in patients treated every 2 weeks and 4 weeks, respectively). ${ }^{29}$ Ixekizumab was also shown to significantly improve skin-related relationship and sexual difficulties. ${ }^{30}$ Pruritus significantly impacted the QoL of patients with psoriasis. ${ }^{31}$ In Uncover-2 and -3, itching severity was measured using an 11-point Numerical Rating Scale (NRS). The proportion of patients who experienced a $\geq 4$-point improvement in NRS and who also had a baseline score of $\geq 4$ points were $85.1 \%$ and $82.5 \%$ in Uncover-2 and -3 , respectively, after 12 weeks when treated with ixekizumab every 2 weeks. The median time to at least a 4-point reduction in ixekizumab-treated patients was 2.1 weeks versus 8.1 weeks in patients treated with etanercept. ${ }^{29}$

\section{Autoantibody formation}

In the Uncover trials, patients were monitored for development of antidrug antibodies (ADAs) that have been known to reduce the efficacy of some biological agents in the past. ${ }^{32}$ Antidrug antibodies were detected using an affinity capture elution approach. ${ }^{33}$ After the 12-week induction period, $91.0 \%$ of patients treated every 2 weeks and $86.6 \%$ of patients treated every 4 weeks were negative for ADAs. Patients who developed ADAs were divided into low $(<1: 160)$, moderate $(\geq 1: 160$ to $<1: 1280)$, and high $(\geq 1: 1280)$ titer groups. At 12 weeks of treatment in patients treated with ixekizumab every 2 and every 4 weeks, respectively, most patients who 
developed ADAs had low titers (5.7\% and 8.0\%), while $1.6 \%$ and $3.0 \%$ patients had moderate titers and $1.7 \%$ and $2.4 \%$ patients had high titers. Patients with high titers had lower PASI response rates compared to patients without ADAs. For example, PASI-75 among patients with high ADA titers was $36 \%$, while in those without ADAs it was $90.7 \% .{ }^{15}$ After 60 weeks of treatment, patients who were treated initially with ixekizumab every 2 weeks followed by maintenance with every 4-week dosing had negative ADAs (84.8\%), low ADA titer $(14.2 \%)$, or moderate ADA titer $(0.9 \%)$; no patients had high titers. ADA levels did not affect maintenance of response through 60 weeks in patients who initially responded after 12 weeks of induction dosing. ${ }^{34}$

\section{Adverse events}

Pooled results from the pivotal Phase III trials showed that, at 12 weeks, patients receiving $80 \mathrm{mg}$ of ixekizumab every 2 weeks experienced adverse events and infections $(54.8 \%$ and $26.9 \%$, respectively) at higher rates than patients treated with placebo (46.8\% and $22.8 \%$, respectively). Injection site reactions, upper respiratory tract infections, nausea, and tinea infections all occurred in patients treated every 2 weeks with ixekizumab $80 \mathrm{mg}$ through 12 weeks at rates higher than $1 \%$ and higher than in patients treated with etanercept or placebo. $^{3}$

Given that ixekizumab targets IL-17, which plays a role in host defense against Candida infections; the rate of Candida infections is of particular interest. In all, 1.4\% of patients receiving ixekizumab every 2 weeks developed Candida infections in the first 12 weeks of treatment, while $0.5 \%$ of patients receiving placebo developed Candida infections in the same time period. ${ }^{35}$ None of these Candida infections met criteria for severe adverse event, and most were oral candidiasis, although vulvovaginal, skin, and esophageal candidiasis did occur in patients who had any exposure to ixekizumab through 60 weeks in pooled date from the Uncover trials. ${ }^{15}$

Patients in clinical trials treated with ixekizumab had higher rates of onset or exacerbation of inflammatory bowel diseases including Crohn's disease (CD; 0.1\%) and ulcerative colitis (UC; $0.2 \%)$ than patients treated with placebo $(0 \%)$ in the 12-week induction period. ${ }^{3}$ Eleven patients reported inflammatory bowel disease between weeks 0 and 60, with seven cases of UC and four cases of CD. Of the seven patients reporting UC, four had a prior history of UC and five had colonoscopies to confirm the diagnosis of UC (the two patients who did not undergo colonoscopy had a prior history of the disease). All four cases of CD were confirmed with colonoscopy. ${ }^{15}$

Using integrated analysis sets from ixekizumab clinical trials, Strober et $\mathrm{al}^{36}$ evaluated both short- and long-term safety outcomes. This dataset included 4209 patients with a total exposure to ixekizumab of 6480 patient-years. The data were split into the 12 -week induction period and 12- to 60-week maintenance period, as well as being presented for all patients treated with ixekizumab across seven clinical trials. Treatment emergent adverse event (TEAE) rates were measured in exposure-adjusted incidence rates (IRs), which were defined as the number of patients with a particular adverse event per 100 patient-years. In the induction period, the IR of infections was significantly higher in ixekizumab-treated patients than in etanercept patients (112.5 in patients treated every 2 weeks vs 93.9 in patients treated with etanercept). However, the IR of particular infection types was not significantly different between ixekizumab- and etanercept-treated patients. The highest IRs were for nasopharyngitis, upper respiratory tract infections, and urinary tract infections in both ixekizumaband etanercept-treated patients. Injection site reaction rates were similar between patients treated with ixekizumab and etanercept, although injection site pain was more common in patients treated with ixekizumab, while injection site erythema was more common in etanercept-treated patients. Other common TEAEs observed in the 12-week induction period included gastrointestinal disorders, musculoskeletal and connective tissue disorders, and headache, all of which were not significantly more common in patients treated with ixekizumab versus etanercept-treated patients. No serious adverse events were significantly more common in ixekizumab-treated patients than in placebo or etanercepttreated patients.

In the maintenance period from week 12 to 60 , patients were treated with ixekizumab at the FDA-recommended frequency of every 4 weeks. In this group of patients, the only significantly increased TEAE was injection site reactions, with an IR of 7.8 per 100 patient-years in ixekizumabtreated patients versus 1.1 per 100 patient-years in patients treated with placebo. The IRs of infections, nasopharyngitis, upper respiratory tract infection, sinusitis, influenza, urinary tract infection, pharyngitis, gastroenteritis, tinea pedis, folliculitis, and oral candidiasis were not significantly greater in patients treated with ixekizumab compared to those treated with placebo. Overall, ixekizumab was very well tolerated. 


\section{Precautions}

Prior to initiating therapy with ixekizumab, patients should be screened for tuberculosis (TB) infection. Patients with active TB infection should not be administered ixekizumab, while patients with latent TB should have treatment initiated prior to starting on ixekizumab.

Ixekizumab is absolutely contraindicated for treatment of patients who have had prior severe hypersensitivity reactions to ixekizumab or any of the excipients found in each syringe or auto-injector.

\section{Conclusion}

Ixekizumab is one of the newest therapies available for the treatment of moderate-to-severe plaque psoriasis, which targets IL-17A, a key mediator in the pathogenesis of psoriasis. Ixekizumab has proven to be one of the most efficacious therapies, with $87.4 \%-89.7 \%$ of patients achieving PASI-75 at week 12 and up to $55 \%$ of patients achieving PASI-100 at week 60. Ixekizumab has been shown to be effective in the treatment of other types of psoriasis, including palmoplantar, erythrodermic, and generalized pustular as well as nails affected by psoriasis. Ixekizumab has also shown to improve QoL in patients with moderate-to-severe psoriasis. ADAs in high titers develop in a very small proportion of patients and may correlate with decreased efficacy of ixekizumab. Ixekizumab is well tolerated by patients, with the most common adverse events being injection site reaction and infections such as nasopharyngitis and upper respiratory tract infections. Further research, including long-term studies in a large population of patients in the real-world setting, is needed to better understand the long-term efficacy and safety of ixekizumab.

\section{Disclosure}

The authors report no conflicts of interest in this work.

\section{References}

1. Drake LA, Ceilley RI, Cornelison RL, et al. Guidelines of care for psoriasis. J Am Acad Dermatol. 1994;28:632-637.

2. Danielsen K, Olsen AO, Wilsgaard T, Furberg AS. Is the prevalence of psoriasis increasing? A 30-year follow-up of a population-based cohort. Br J Dermatol. 2013;168:1303-1310.

3. Taltz [package insert]. Eli Lilly and Company, Indianapolis, IN; 2016.

4. Di cesare A, Di meglio P, Nestle FO. The IL-23/Th17 axis in the immunopathogenesis of psoriasis. J Invest Dermatol. 2009;129(6): 1339-1350.

5. Iwakura Y, Ishigame H. The IL-23/IL-17 axis in inflammation. J Clin Invest. 2006;116(5):1218-1222.

6. Lowes MA, Suárez-fariñas M, Krueger JG. Immunology of psoriasis. Annu Rev Immunol. 2014;32:227-255.
7. Lowes MA, Kikuchi T, Fuentes-duculan J, et al. Psoriasis vulgaris lesions contain discrete populations of Th1 and Th17 T cells. J Invest Dermatol. 2008;128(5):1207-1211.

8. Raychaudhuri SP. Role of IL-17 in psoriasis and psoriatic arthritis. Clin Rev Allergy Immunol. 2013;44(2):183-193.

9. Ariza ME, Williams MV, Wong HK. Targeting IL-17 in psoriasis: from cutaneous immunobiology to clinical application. Clin Immunol. 2013;146(2):131-139.

10. Martin DA, Towne JE, Kricorian G, et al. The emerging role of IL-17 in the pathogenesis of psoriasis: preclinical and clinical findings. J Invest Dermatol. 2013;133(1):17-26.

11. Weaver CT, Hatton RD, Mangan PR, Harrington LE. IL-17 family cytokines and the expanding diversity of effector T cell lineages. Annu Rev Immunol. 2007;25:821-852.

12. Gaffen SL. Structure and signalling in the IL-17 receptor family. Nat Rev Immunol. 2009;9(8):556-567.

13. Giunta A, Ventura A, Chimenti MS, Bianchi L, Esposito M. Spotlight on ixekizumab for the treatment of moderate-to-severe plaque psoriasis: design, development, and use in therapy. Drug Des Devel Ther. 2017;11:1643-1651.

14. Krueger JG, Fretzin S, Suárez-fariñas M, et al. IL-17A is essential for cell activation and inflammatory gene circuits in subjects with psoriasis. J Allergy Clin Immunol. 2012;130(1):145.e9-154.e9.

15. Gordon KB, Blauvelt A, Papp KA, et al. Phase 3 trials of ixekizumab in moderate-to-severe plaque psoriasis. $N$ Engl J Med. 2016;375(4):345-356.

16. Griffiths CE, Reich K, Lebwohl M, et al. Comparison of ixekizumab with etanercept or placebo in moderate-to-severe psoriasis (UNCOVER-2 and UNCOVER-3): results from two phase 3 randomised trials. Lancet. 2015;386(9993):541-551.

17. Syed YY. Ixekizumab: a review in moderate to severe plaque psoriasis. Am J Clin Dermatol. 2017;18(1):147-158.

18. Blauvelt A, Papp KA, Sofen H, et al. Continuous dosing versus interrupted therapy with ixekizumab: an integrated analysis of two phase 3 trials in psoriasis. J Eur Acad Dermatol Venereol. 2017;31(6):1004-1013.

19. Reich K, Puig L, Mallbris L, Zhang L, Osuntokun O, Leonardi C. The effect of bodyweight on the efficacy and safety of ixekizumab: results from an integrated database of three randomised, controlled phase 3 studies of patients with moderate-to-severe plaque psoriasis. J Eur Acad Dermatol Venereol. 2017;31(7):1196-1207.

20. Gottlieb AB, Lacour JP, Korman N, et al. Treatment outcomes with ixekizumab in patients with moderate-to-severe psoriasis who have or have not received prior biological therapies: an integrated analysis of two phase III randomized studies. J Eur Acad Dermatol Venereol. 2017;31(4):679-685.

21. Reich K, Pinter A, Lacour JP, et al. Comparison of ixekizumab with ustekinumab in moderate-to-severe psoriasis: 24-week results from IXORA-S, a phase III study. Br J Dermatol. Epub 2017 May 19.

22. Menter A, Warren RB, Langley RG, et al. Efficacy of ixekizumab compared to etanercept and placebo in patients with moderate to severe plaque psoriasis and non-pustular palmoplantar involvement: results from three phase 3 trials (UNCOVER-1, -2, and -3). J Eur Acad Dermatol Venereol. Epub 2017 Mar 21.

23. Van de kerkhof P, Guenther L, Gottlieb AB, et al. Ixekizumab treatment improves fingernail psoriasis in patients with moderate-to-severe psoriasis: results from the randomized, controlled and open-label phases of UNCOVER-3. J Eur Acad Dermatol Venereol. 2017;31(3):477-482.

24. Saeki H, Nakagawa H, Ishii T, et al. Efficacy and safety of open-label ixekizumab treatment in Japanese patients with moderate-to-severe plaque psoriasis, erythrodermic psoriasis and generalized pustular psoriasis. J Eur Acad Dermatol Venereol. 2015;29(6):1148-1155.

25. Saeki H, Nakagawa H, Nakajo K, et al. Efficacy and safety of ixekizumab treatment for Japanese patients with moderate to severe plaque psoriasis, erythrodermic psoriasis and generalized pustular psoriasis: results from a 52-week, open-label, phase 3 study (UNCOVER-J). J Dermatol. 2017;44(4):355-362. 
26. Leonardi C, Matheson R, Zachariae C, et al. Anti-interleukin-17 monoclonal antibody ixekizumab in chronic plaque psoriasis. $N$ Engl J Med. 2012;366(13):1190-1199.

27. Gordon KB, Leonardi CL, Lebwohl M, et al. A 52-week, open-label study of the efficacy and safety of ixekizumab, an anti-interleukin-17A monoclonal antibody, in patients with chronic plaque psoriasis. $\mathrm{J} \mathrm{Am}$ Acad Dermatol. 2014;71(6):1176-1182.

28. Langley RG, Rich P, Menter A, et al. Improvement of scalp and nail lesions with ixekizumab in a phase 2 trial in patients with chronic plaque psoriasis. J Eur Acad Dermatol Venereol. 2015;29(9):1763-1770.

29. Leonardi CL, Blauvelt A, Sofen HL, et al. Rapid improvements in healthrelated quality of life and itch with ixekizumab treatment in randomized phase 3 trials: results from UNCOVER-2 and UNCOVER-3. J Eur Acad Dermatol Venereol. 2017;31(9):1483-1490.

30. Guenther L, Warren RB, Cather JC, et al. Impact of ixekizumab treatment on skin-related personal relationship difficulties in moderateto-severe psoriasis patients: 12 week results from two Phase 3 trials. $J$ Eur Acad Dermatol Venereol. Epub 2017 Jun 2.

31. Reich A, Hrehorów E, Szepietowski JC. Pruritus is an important factor negatively influencing the well-being of psoriatic patients. Acta Derm Venereol. 2010;90(3):257-263.
32. Hsu L, Snodgrass BT, Armstrong AW. Antidrug antibodies in psoriasis: a systematic review. Br J Dermatol. 2014;170(2):261-273.

33. Muram TSJ, Chain J, Komocsar W, et al. P87: a highly sensitive and drug-tolerant immunogenicity screening assay for ixekizumab, an antiinterleukin-17A antibody, using an affinity capture elution approach. 7th International Congress of Psoriasis: from Gene to Clinic. 18 December 2014 ed. The Queen Elizabeth II Conference Centre, London, UK. 11th-13th December 2014. Br J Dermatol. 2014:e105-e173.

34. Blauvelt A, Cameron G, Gordon K, et al. Ixekizumab, a novel anti-IL17A antibody, exhibits low immunogenicity during long-term treatment in patients with psoriasis [abstract no. 3232]. $\mathrm{J} \mathrm{Am} \mathrm{Acad} \mathrm{Dermatol.}$ 2016;74(5 Suppl 1):AB258.

35. Farahnik B, Beroukhim K, Zhu TH, et al. Ixekizumab for the treatment of psoriasis: a review of phase III trials. Dermatol Ther (Heidelb). 2016;6(1):25-37.

36. Strober B, Leonardi C, Papp KA, et al. Short- and long-term safety outcomes with ixekizumab from 7 clinical trials in psoriasis: etanercept comparisons and integrated data. JAm Acad Dermatol. 2017;76(3):432. e17-440.e17.
Psoriasis: Targets and Therapy

\section{Publish your work in this journal}

Psoriasis: Targets and Therapy is international, peer-reviewed, open access journal focusing on psoriasis, nail psoriasis, psoriatic arthritis and related conditions, identification of therapeutic targets and the optimal use of integrated treatment interventions to achieve improved outcomes

\section{Dovepress}

and quality of life. Visit http://www.dovepress.com/testimonials.php to read real quotes from published authors. 